\title{
Prototipo de plataforma para vigilancia de inmuebles rurales mediante computación en la nube y supervisión con drones
}

\author{
Platform Prototype for Surveilling Rural Properties \\ Using Cloud Computing and Surveillance Drones
}

\author{
Maycol Alexander Segura-Cuervo ${ }^{1} \bowtie$, Jairo Alonso Mesa Lara ${ }^{2}$
}

' Universidad Pedagógica y Tecnológica de Colombia, Tunja

${ }^{2}$ Universidad Pedagógica y Tecnológica de Colombia, Tunja

Avenida central del norte 39-115, Edificio central, oficina C-325, Escuela de Ingeniería de Sistemas y Computación, Universidad Pedagógica Tecnológica de Colombia, Tunja, Colombia. Email: maycol.segura@uptc.edu.co

Recibido: 10 de agosto del 2017 Aprobado: 30 de noviembre del 2017 Disponible en línea: 1 de enero del 2018

How to cite this article: M. A. Segura-Cuervo y J. A. Mesa-Lara, "Prototipo de plataforma para vigilancia de inmuebles rurales usando computación en la nube y supervisión con drones", Revista Ingeniería Solidaria, vol. 14, no. 24, pp. 17, enero 2018. doi: https://doi.org/10.16925/in.v14i24.2160

\section{Resumen}

Introducción: el artículo es el resultado de la investigación "Sistema de monitoreo de alarmas basado en vehículos aéreos no tripulados" desarrollada en la Universidad Pedagógica y Tecnológica de Colombia durante el 2016. Muestra las fases de construcción del prototipo de plataforma de monitoreo de alarmas electrónicas con la utilización de la computación en la nube, apoyado en la supervisión a través de drones.

Objetivo: mejorar los tiempos de respuesta en la supervisión a inmuebles rurales ante una señal de alerta al recopilar con rapidez evidencias externas captadas en fotos y videos ante posibles casos de hurto. Metodología: se identificó la forma de prestación del servicio de monitoreo en Colombia y luego se diseñaron e implementaron cinco módulos: intrusión, recepción, administración, monitoreo y supervisión. Resultados: una vez alterada una variable de intrusión y detectada por los sensores, el sistema de alarma trasmitió la señal de alerta a través de comunicación IP/GPRS a la receptora de señales en la nube, la cual traduce el mensaje y lo direcciona al software de administración encargado de enviar el plan de vuelo y las coordenadas geográficas requeridas para realizar la supervisión con un dron en sitio.

Conclusión: se logró calcular los tiempos de respuesta en la supervisión con drones, de manera que se encontró una respuesta favorable para el servicio de monitoreo y se desarrolló el software de administración.

Originalidad: obtuvo una plataforma con elementos innovadores asociados a la integración entre drones y computación en la nube.

Limitaciones: la regulación colombiana sobre drones restringe los vuelos autónomos.

Palabras clave: alarmas, drones, monitoreo, seguridad, seguridad, sensores. 


\title{
Platform Prototype for Surveilling Rural Properties Using Cloud Computing and Surveillance Drones
}

\author{
Abstract \\ Introduction: The article derives from the research "Alarm monitoring system based on unmanned aerial \\ vehicles" conducted at Universidad Pedagógica y Tecnológica de Colombia in 2016. It shows the construc- \\ tion phases of an electronic alarm monitoring platform prototype using cloud computing, supported by \\ surveillance drones. \\ Aim: To improve response times in the surveillance of rural properties upon a warning signal of possible theft \\ by quickly collecting external evidence captured in photos and videos. \\ Methods: We identified how the monitoring service is provided in Colombia and then five modules were \\ designed and implemented: intrusion, reception, management, monitoring and surveillance. \\ Results: Once an intrusion variable was altered and detected by the sensors, the alarm system transmitted \\ the warning signal through IP/GPRS communication to the signal receiver in the cloud, which translates the \\ message and directs it to the management software responsible for sending the flight plan and geographic \\ coordinates required for onsite drone surveillance. \\ Conclusion: Response times on drone surveillance could be calculated with favorable results for the moni- \\ toring service, and the management software was developed. \\ Originality: We obtained a platform with innovative elements associated with the integration of drones and \\ cloud computing. \\ Limitations: Colombian regulations on drones restrict unmanned flights.
}

Keywords: alarms, drones, monitoring, security, sensors.

\section{Protótipo de plataforma para vigilância de imóveis rurais mediante computação em nuvem e supervisão com drones}

\begin{abstract}
Resumo
Introdução: este artigo é o resultado da pesquisa "Sistema de monitoramento de alarmes baseado em veículos aéreos não tripulados", desenvolvida na Universidad Pedagógica y Tecnológica de Colombia, durante 2016. Mostra as fases de construção do protótipo de plataforma de monitoramento de alarmes eletrônicos com a utilização da computação em nuvem, apoiado na supervisão por meio de drones.

Objetivo: melhorar os tempos de resposta na supervisão de imóveis rurais ante um sinal de alerta ao recopilar com rapidez evidências externas captadas em fotos e vídeos diante de possível casos de furto.
\end{abstract}

Metodologia: identificou-se a forma de prestação do serviço de monitoramento na Colômbia e, logo, desenharam-se e implementaram-se cinco módulos: intrusão, recepção, administração, monitoramento e supervisão.

Resultados: uma vez alterada uma variável de intrusão e detectada pelos sensores, o sistema de alarme transmitiu 0 sinal de alerta por meio de comunicação IP/GPRS à receptora de sinais na nuvem, a qual traduz a mensagem e direciona-a ao software de administração encarregado de enviar o plano de voo e as coordenadas geográficas requeridas para realizar a supervisão com um drone.

Conclusão: pôde-se calcular os tempos de resposta na supervisão com drones, de maneira que se encontrou uma resposta favorável para o serviço de monitoramento e desenvolveu-se 0 software de administração.

Originalidade: obteve-se plataforma com elementos inovadores associados à integração entre drones e computação em nuvem.

Limitações: a regulamentação colombiana sobre drones restringe os voos autônomos.

Palavras-chave: alarmes, drones, monitoramento, segurança, sensores. 


\section{Introducción}

El monitoreo de alarmas es un servicio empleado con el fin de vigilar inmuebles físicos ante posibles actos de intrusión sin permiso. Consiste en la instalación de sensores, paneles y comunicadores electrónicos para la vigilancia de propiedades físicas de forma remota. Estos sistemas emiten señales

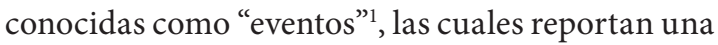
emergencia o una condición del sistema a una central de monitoreo que está a cargo de personal las 24 horas del día y supervisa la recepción de señales para darles tramite según sea la necesidad.

El sistema desarrollado se ajusta dependiendo del tipo de emergencia, se actúa con supervisión motorizada y, en caso de necesidad, se da aviso a las autoridades bien sea la Policía, los Bomberos o bien médicos, y así acudan al lugar de los hechos.

Estos servicios están a cargo de empresas de seguridad sin armas conocidas también como "empresas de monitoreo o seguridad electrónica”. En Colombia, existen 79 empresas habilitadas y licenciadas para prestar este servicio, según reporte de la Superintendencia de Vigilancia y Seguridad Privada con corte al 25 de julio del 2017 [1].

El monitoreo de alarmas es conocido principalmente por su actividad en zonas urbanas, sin embargo, en zonas rurales también se presta el servicio en empresas y casas. Existen dos problemáticas principales en la prestación del servicio en zonas rurales: la primera es una tardía presencia en sitio por parte de los supervisores, especialmente por aspectos de desplazamiento y la segunda es la poca inclusión en el servicio de aplicaciones remotas para la activación y desactivación de dispositivos que ayuden a disuadir o reducir los riesgos de un robo.

La supervisión en inmuebles en zonas rurales representa una tarea pendiente por resolver, debido a que existen problemáticas en Colombia en cuanto a veredas con vías angostas, sin pavimentar en su mayoría, con difícil acceso para vehículos y en algunos casos quedan distantes de las cabeceras urbanas; a esto se le suman variables como condiciones ambientales y bajas velocidades en los automotores por el estado de las carreteras. Frente al modelo tradicional de seguridad todas estas va-

Señales enviadas por la alarma o datos formato Contact ID. riables protagonizan una dificultad en la prestación del servicio de monitoreo de alarma por cuanto no se puede cumplir a satisfacción la supervisión cuando ocurran situaciones de emergencias ya que estas se atenderían con elevados tiempos de respuesta, ofreciendo un servicio deficiente en una de sus etapas claves de reacción y salvaguarda [2].

Por otra parte:

La Policía Nacional registró 678.958 delitos en la zona urbana y 97.490 en la rural; los departamentos con mayor registro en esta última zona son Antioquia, con 11.995 delitos, y Valle, con 11.660. Las 32 ciudades capitales del país registraron el 56,16\% (437.953) del total de los delitos establecidos en el 2015; Bogotá registró el 14,64 \% (114.181), Medellín el 7,23 \% (56.391) y Cali el 5,69 \% (44.405) de los delitos [3].

Según registros dela criminalidad en Colombia y la actividad operativa de la Policía Nacional:

En el año 2015 se registraron 21.139 casos de hurto a residencias. La ciudad capital y cinco departamentos representaron el 53,73 \% del total de los casos: Bogotá (17,14\%), Antioquia (12,01\%), Valle $(7,97 \%)$, Meta $(6,01 \%)$, Tolima $(5,96 \%)$ y Santander (4,64\%). Asimismo, se registraron 22.455 casos de hurto a entidades comerciales; la ciudad de Bogotá (26,37 \%) y el departamento de Antioquia $(14,99 \%)$ representaron el $41,36 \%$ del total de los registros [3].

También se presenta en zonas rurales de Colombia el tipo de hurto conocido como "abigeato":

El abigeato es un delito punible y estipulado en muchos códigos legales de la mayoría de los países ganaderos; consiste en el robo o hurto de ganado o animales domésticos, principalmente caballos y vacas, aunque también se da en ovinos. Según el Observatorio de Derechos Humanos y el Derecho Internacional Humanitario de la Fundación Colombia Ganadera (Fundagán) y la Dirección de Carabineros y Seguridad Rural, en 2014 se registraron 164 casos de abigeato, los cuales dejaron pérdidas por más de 15.000 millones de pesos. En lo corrido del año ya son más de 2000 millones. La insegu- 
ridad en los predios ganaderos da Colombia cada día aumenta más. Departamentos como Huila, La Guajira, Atlántico y Boyacá denuncian constantemente casos de abigeato y hurto de enseres en los hatos bovinos [4].

La plataforma de monitoreo se diseñó para inmuebles en zonas rurales en las que la supervisión se realiza con apoyo de drones. Su diseño opera bajo el entorno de la computación en la nube, la cual presta funcionalidades de software e infraestructura como servicio y se creó para la administración de abonados interconectados con receptoras de eventos a través de puertos serie o $\mathrm{TCP}^{2} / \mathrm{IP}^{3}$. Toda la infraestructura tecnológica se encuentra instalada en servidores en la nube a fin de gestionar un sistema más eficiente con espejos para respaldo de información y continuidad del negocio. El software diseñado para la administración es multiusuario y pueden emplearlo varias empresas al mismo tiempo, e iniciar sesión cada una con sus credenciales de validación, de modo que allí podrán gestionar todos sus clientes.

Entre las características más importantes, se encuentra la posibilidad de incorporar toda la estructura de software y hardware de una central de monitoreo en la nube, así como contar con receptoras físicas y virtuales, además de estar en capacidad de gestionar automáticamente el vuelo de un dron para tareas de supervisión en busca, esencialmente, de brindar una respuesta oportuna en la recolección de evidencias, previa recepción de un evento de alarma de robo.

Los drones hacen parte de la supervisión de cada área y están ubicados estratégicamente en zonas donde se presta el servicio de monitoreo.

En la comunicación se emplean módulos $\mathrm{IP}^{4}$ y módulos GPRS $^{5}$ conectados a la red de telefonía celular.

El levantamiento de información para diseñar la plataforma se realizó mediante encuestas aplicadas a tres empresas con cobertura nacional en las que se evidenciaron dos problemáticas: la primera es la supervisión a zonas rurales, y la segunda la falta de planes de continuidad de negocio en las empresas prestadoras del servicio.

\footnotetext{
Protocolo de control de trasmisión.

Protocolo de Internet.

Comunicación a través del protocolo de Internet.

Servicio general de paquetes vía radio. (GPRS, por sus
} siglas en inglés)

\subsection{Trabajos relacionados}

Monitoreo.com es una plataforma de monitoreo de alarmas y cámaras de seguridad presente en varios países de América: "Presenta un concepto de trabajo en RED en el cual desarrolla el servicio de monitoreo de alarmas desde una perspectiva de innovación, ya que todas sus operaciones se efectúan íntegramente a través de Internet" [5].

Se basa en una red de empresas asociadas que colaboran mutuamente a través de la plataforma de monitoreo $100 \%$ en la nube, usa servidores Linux a través de Internet, web 2.0, ofrece back up, redundancia en sus servicios y recepción de eventos en protocolos contact $\operatorname{ID}^{6} \mathrm{y} 4+2,{ }^{7}$ lo cual permite la conexión de varias marcas de alarma y comunicadores a través de línea telefónica, GPRS ${ }^{8}$ e IP $P^{9}$. Dentro de sus características importantes, se cuenta la inclusión de la domótica y el uso de aplicaciones móviles para sus usuarios finales.

Por otro lado, Softguard es el líder del software de monitoreo. Según la empresa Keitech Colombia:

Cuenta con una interfaz usable y ágil, que permite la automatización de procesos permitiendo a operadores enfocarse en lo prioritario o importante. SoftGuard es el software de monitoreo de alarmas $y$ video integral que evoluciona su empresa y sus servicios y le permite ser líder en el negocio. Ha sido reconocido por ser el de mayor crecimiento en América, con más de 3900 licencias de módulos en sus 11 años de vida [6].

El software de monitoreo integra servicios de monitoreo de alarmas, videovigilancia, rastreo vehicular, monitoreo humano y control de rondas, entre otros. Por ser un software propietario, se pone a disposición de las empresas de monitoreo a través de licencias de uso y se instala en la infraestructura de cada empresa.

Al revisar implementaciones de sistemas de seguridad en otros países como Chile, Peillard menciona que existen fórmulas de regulación y debates que apuntan, principalmente, a comprender la utilidad del monitoreo electrónico [7].

\footnotetext{
6 Protocolo de comunicación universal empleado en sistemas de seguridad.

7 Protocolo de comunicación de alarmas.

8 Servicio general de paquetes vía radio.

9 Protocolo de Internet.
} 
En China, se presentó un sistema basado en tecnología integrada con comunicación inalámbrica GPRS, sistema de mensajería multimedia y control remoto para supervisión en tiempo real en un sistema de alarma. Este sistema muestra sus principales funciones de la siguiente manera: cuando una persona entra ilegalmente en un espacio físico ocurre un disparo por sensores infrarrojos piroeléctricos y la cámara de forma automática entra en escena y captura imágenes; se transfiere entonces el disparo al procesador integrado del módulo para, posteriormente, enviar reportes vía mensaje de texto o transferir la alerta con las imágenes adjuntas a través de Internet [8].

Según [9], los sistemas de alarma no pueden enfrentar las amenazas de seguridad de manera satisfactoria. A fin de combatir este problema, se desarrolló un sistema de alarma de alta confianza que establece la conexión de Internet, es decir, un $\mathrm{TCP}^{10} / \mathrm{IP}$ a través de GPRs/CDMA ${ }^{11} / 3 \mathrm{G}^{12}$. Así, se logra el control de comunicación entre terminales, interfaces hombre-máquina y los usuarios por medio de la actual red de comunicaciones móviles. El sistema se ha aplicado con éxito en la práctica y los resultados muestran que el sistema cumple con los requerimientos de seguridad.

En Ecuador, Moreno desarrolló un trabajo de investigación en el que se estudiaron los sistemas de monitoreo y vigilancia del área agrícola de Tulcán. Se realizó una investigación de campo con la aplicación de entrevistas y encuestas a personas que trabajan en las fincas del sector rural con el propósito de identificar si los pobladores de estas zonas permitían el sobrevuelo de drones para el monitoreo y la identificación de variables a fin de mejorar los cultivos en sus fincas [10].

En España, Galdon presenta un debate con una perspectiva sociológica y politológica basado en el uso de las cámaras de seguridad, y en torno al cual el autor formula preguntas de interés para desarrollar el trabajo: ¿por qué la videovigilancia? ¿Cómo es posible que la videovigilancia haya conseguido no solo imponerse sino seguir una trayectoria de expansión, mantener cifras de apoyo popular considerables y generar un consenso favorable inquebrantable entre los responsables políticos, las autoridades policiales y la población en

\footnotetext{
10 Protocolo de control de trasmisión.

11 Acceso múltiple por división de código.

12 Tercera generación de transmisión de voz y datos a través de telefonía móvil.
}

general? El artículo trata el tema de las cámaras de seguridad y de cómo la videovigilancia hace varios años invade las aplicaciones de seguridad en todo el mundo, y en especial en España [11]. Sumado a esto, el Ministerio de Defensa de España ha realizado un análisis sobre drones al evaluar diferentes características tales como generalidades, misión, capacidades, certificación, integración en el espacio aéreo, entrenamiento, necesidad estratégica industrial, estado y tendencia [12].

En Colombia, se desarrolló el proyecto "Ingeniería básica para el diseño sistema de monitoreo, detección y alarma, humo, fuego y gas (F\&G) de las estaciones de compresión de gas de la Trasportadora de Gas Internacional TGI". En este se presenta la ingeniería básica del sistema de monitoreo, detección y alarma del sistema de humo, fuego y gas para las estaciones de compresión que hacen parte del gasoducto Cusiana-Ballenas, en conformidad con los lineamientos de la norma NFPA-72 [13].

Adicionalmente, en Colombia, Jochen Kleinschmidt expone la creciente utilización de sistemas aéreos no tripulados y sus operaciones en guerras. Debido a sus diferentes aplicaciones se referencia un cambio evolutivo en el ordenamiento espacial de la política global y su normatividad. Como aporte al proyecto planteado, se identifica la discusión sobre asuntos de responsabilidad legal o moral, individual o colectiva en el uso de la nueva tecnología y de qué manera afecta esto a las personas [14].

En Nueva Zelanda, Christensen observó la aparición de una tecnología potencial de detección remota y aviones dirigidos por control remoto con sistema de visión térmica/infrarroja con base en un análisis de costo-beneficio. La metodología que utilizó presenta un enfoque basado en la encuesta en línea dirigido a expertos en la materia en la gestión de los incendios forestales y de aviones no tripulados. A fin de evaluar, se utilizó el cambio porcentual en la efectividad de los cinco diferentes escenarios de gestión con aviones piloteados a distancia y un sistema de visión térmica/infrarroja [15].

En Estados Unidos de América, existen estudios sobre la creciente iniciativa del dron para detecciones remotas y sus ventajas en la investigación de prácticas en la ingeniería forestal. Se analizan los diversos enfoques del dron en detección remota para los bosques al ubicar los espacios de follaje, medir la altura del follaje forestal y hacer seguimiento de los incendios forestales. En la investigación se describen algunos beneficios de los drones en detección remota, lo que incluye materiales, 
costos de operación, control flexible de la resolución espacial y temporal, la recopilación de datos de alta intensidad y la ausencia de riesgo para las tripulaciones [16].

A nivel mundial, varios autores han proyectado trabajos sobre la problemática del uso de vehículos aéreos no tripulados en cuanto a su energía inicial limitada y el consumo, relacionada con la altitud para las zonas que necesiten mayor vista panorámica en su monitoreo [17]. También se encuentran escritos sobre el impacto y la incursión del dron en varias áreas socioeconómicas tales como el transporte, la comunicación, la agricultura, la mitigación de desastres, la preservación del medio ambiente y la vigilancia [18]. En muchos países, se desarrolla el debate sobre el uso doméstico de los drones generalizado para el sector de la vigilancia, y sobre cómo los drones erosionarán la privacidad individual de las personas [19]. Existen proyectos académicos basados en investigaciones y desarrollos dirigidos a lograr el diseño y la implementación de una computadora de vuelo para aeronaves no tripuladas. La idea surge del estudio de dos ramas de la ingeniería electrónica: la teoría de control y los sistemas embebidos [20].

Zahawi et al. presentan un documento que evalúa si las mediciones de teledetección desde vehículos aéreos no tripulados de peso ligero son un sustituto rentable para medidas de campo tradicionales de acuerdo con el volumen de datos que se puede generar en un solo plan de vuelo, así como la capacidad de utilizar la tecnología en áreas remotas [21].

Por su parte, entidades como la Organización de Aviación Civil Internacional proporcionan un marco normativo internacional mediante normas y métodos recomendados para drones, con el apoyo de procedimientos dirigidos a los servicios de navegación aérea y textos de orientación, con el fin de afianzar la operación normal en todo el mundo de forma segura, armonizada y fluida, comparable a la de las operaciones tripuladas [22].

\section{Metodología}

Se identificó la forma de prestación del servicio de monitoreo en Colombia y luego se diseñaron e implementaron cinco módulos: intrusión, recepción, administración, monitoreo y supervisión.

\subsection{Descripción del diseño de la plataforma de vigilancia}

La plataforma diseñada se basa en un modelo de negocio para el monitoreo de alarmas con la integración de drones. Su objetivo es reducir tiempos de respuesta en la supervisión con base en los altos índices de robo en zonas rurales. Incluye sistemas de alarmas profesionales homologados por la Unión Europea y el Gobierno canadiense, así como drones creados por empresas extranjeras. Como aporte a la plataforma propuesta, se desarrolló el software administrador de eventos, el cual permite la interacción entre sistemas de alarma y la supervisión automática con el dron. Todo el sistema software se diseñó con el fin de que opere en la nube, con lo cual se creó una central de monitoreo accesible a cualquier empresa en el mundo.

\subsubsection{Módulo 1: sistema de intrusión}

En la figura 1, se representa un inmueble monitoreado que contiene un sistema de alarma de intrusión equipado con un arreglo de sensores infrarrojos de movimiento, panel central, alimentación eléctrica, sirena y módulos de comunicaciones $\mathrm{GPRS}^{13} / \mathrm{IP}^{14}$. Una vez los sensores del sistema de alarma detectan una violación, se envía un evento en protocolo Contact $\mathrm{ID}^{15}$ a la receptora de eventos en nube a través de módulos de comunicación IP o GPRS. La plataforma es capaz de recibir todos los tipos de eventos de alarma exitentes en protocolo Contact ID [23]. Toda la comunicación se realiza mediante el uso del servicio de Internet ofrecido por operadores externos. Actualmente, la receptora está parametrizada para recepcionar eventos de comunicadores PCs $250^{16}$ e IP $150^{17}$ Paradox $^{18}$, $\mathrm{LX} 20 / \mathrm{LX} 20 \mathrm{~S}^{19} \mathrm{EBS}^{20}, \mathrm{y} \mathrm{GS} 3125^{21} \mathrm{DSC}^{22}$.

\footnotetext{
13 Servicio general de paquetes vía radio.

Protocolo de Internet.

Protocolo de comunicación en sistemas de alarma.

Módulo de comunicación GPRs Paradox.

Módulo de comunicación a través de Internet del fabri-

cante Paradox.

18 Marca fabricante de sistema de alarmas.

19 Módulo de comunicación GPRS universal del fabricante EBS.

20 Marca fabricantes de alarma.

21 Módulo de comunicación GPRS del fabricante dsc.

22 Marca fabricantes de alarma, sistemas digitales de control.
} 


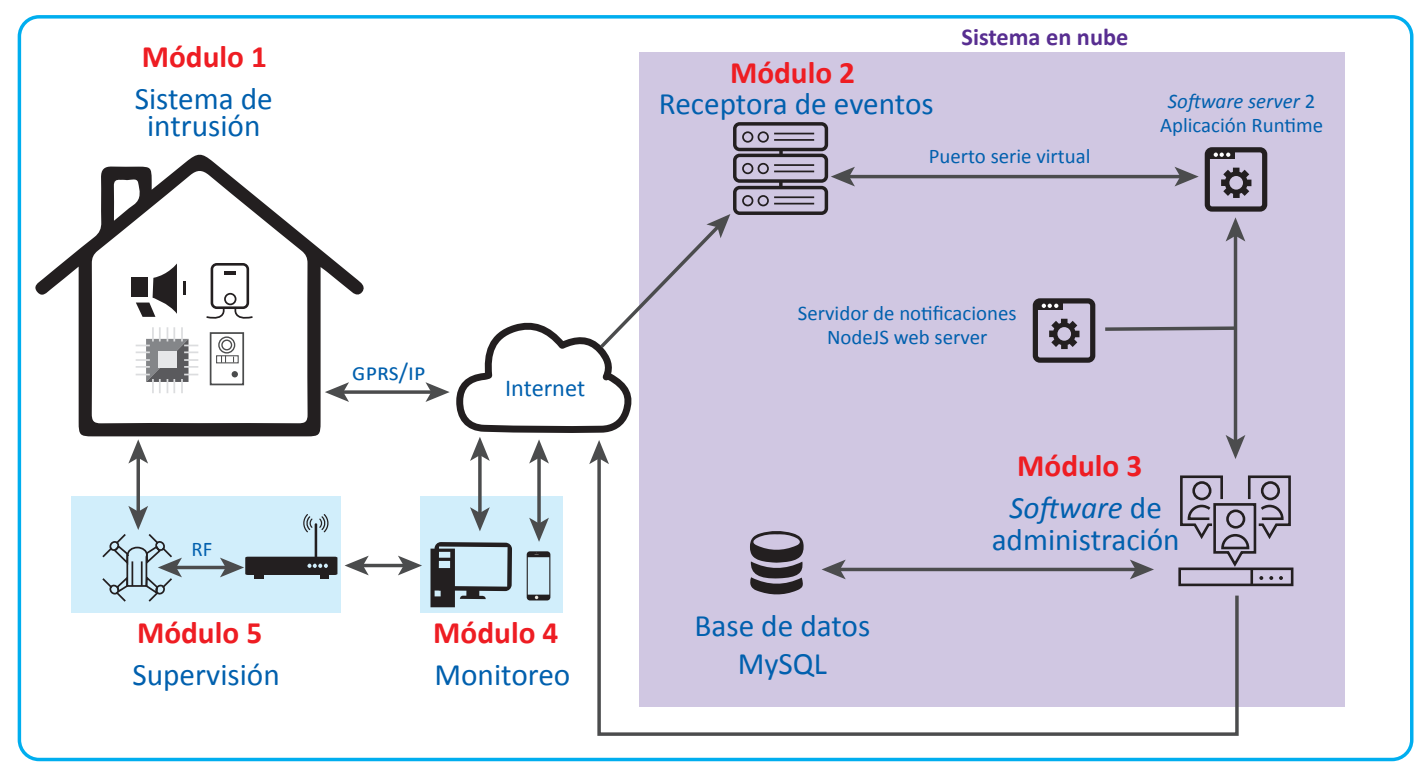

Figura 1. Plataforma de vigilancia rural con supervisión a través de drones Fuente: elaboración propia

\subsubsection{Módulo 2: receptoras de eventos}

Las receptoras virtuales son una herramienta de software que permite simular una receptora física, convierte la señal de la alarma en una cadena conformada por 15 caracteres que contiene el protocolo Contact ID y la envía a un puerto serial virtual; dicho puerto es un programa informático que permite la comunicación entre dos puertos serie. Este puerto serie virtual es el medio para enviar los eventos de la receptora virtual a la aplicación Runtime que tiene por labor leer el puerto serie virtual cada 0,5 segundos, y así tomar registros constantes de los 15 caracteres de la cadena Contact ID con base en una estructura de datos tipo FIFo (por sus siglas en ingles first in first out).

\subsubsection{Módulo 3: software de administración}

Recibe la cadena y la convierte en información legible para el usuario, de manera que obtiene el abonado, la zona, el tipo de evento, etc. Cuenta con un servidor de notificaciones encargado de comunicar todos los eventos en tiempo real a los dispositivos conectados al módulo de monitoreo. Adicionalmente, se diseñó con una base de datos para almacenar la información. Los módulos 2 y 3 se implementan haciendo uso de la computación en la nube con el fin de contar con mayor disponibilidad del servicio y como mecanismo que permite garantizar la continuidad del negocio.

\subsubsection{Módulo 4: monitoreo}

La conexión a la plataforma se efectua a través de Internet desde cualquier explorador web como Google o Mozilla, entre otros. Se realiza con el ingreso a la pagina web de la plataforma, lo que permite a las empresas usuarias ingresar sus credenciales de validación para acceder a la administración de sus abonados/clientes. De forma complementaria, se diseñó una aplicacion móvil con el objetivo de notificar al usuario final sobre las señales que emite su sistema de alarma y su estado en tiempo real.

\subsubsection{Módulo 5: supervisión}

Los vehículos aéreos no tripulados estarán ubicados estratégicamente en cada ciudad, en la zona rural donde se ofrezca el servcio de monitoreo, con el fin de proporcionar supervisión a través de vuelos automáticos, es decir, se determinarán los radios de cobertura y se dispondrán drones en estaciones para un alcance por zona con un radio máximo de ocho kilometros. 


\subsection{Implementación del prototipo}

De acuerdo con encuestas aplicadas a las empresas de monitoreo en Colombia, se identificó que los sistemas de alarmas más utilizados pertenecen a las marcas DSC y Paradox. En la implementación del prototipo, se empleó este tipo de alarmas, el cual permitió validar el funcionamiento del proyecto. Para la recepción de eventos se empleó una receptora virtual IPRS- $7^{23}$ marca Paradox, la cual envía los eventos al sistema administrador. En la supervisión con drones de forma automática, se seleccionó el dron Solo ${ }^{24} 3 \mathrm{DR}^{25}$ por su flexibilidad a la hora de programar acciones automáticas en su plan de vuelo.

En el módulo 1 de la figura 2, se muestra el sistema de alarma con los medios de comunicación y con un sensor infrarrojo pasivo; la alarma es el sistema instalado en la casa de campo. Consta de un panel Paradox referencia SP4000 $0^{26}$ [24], una batería de $12 \mathrm{~V}$ a $7 \mathrm{~A}$, transformador de $110 \mathrm{VAC}$ a 18 VAC, sirena de $30 \mathrm{w}$, un sensor infrarrojo pasivo y un teclado. Para la comunicación, se dispuso de un módulo $\mathrm{GPRS}^{27} \operatorname{PCS}^{2} 00^{28}$ [25], y un módulo IP150 ${ }^{29}$ [26]. La programación del panel y de los módulos de comunicación se realizó con el software Babyware ${ }^{30}$ y se adquirió una tarjeta sim de datos verticales con capacidad de 5 mbps para la conexión a la red GSM.

Los dos medios de comunicación apuntan al módulo 2 en el que se encuentra alojada en la nube la receptora de eventos. La plataforma en nube para la receptora de eventos y el software de administración se instalaron en servidores de la empresa Amazon a través del servicio Aws" "Amazon Web Service" [27]. En el caso de la receptora virtual de eventos se utilizó el software IPRs-732, y se instaló en un servidor con sistema operativo Windows server 2012; a fin de alojar el sistema de administración se utilizó un servidor con sistema operativo Linux.

Al software de administración se le dio el nombre de "Ceven" y se construyó basado en carac-

Receptora virtual de eventos del fabricante Paradox.

Nombre del dron.

Marca de fabricante de drones estadounidense.

Panel principal de alarma de cuatro zonas.

Servicio general de paquetes vía radio.

Módulo de comunicación GPRs del fabricante Paradox.

Módulo de comunicación IP del fabricante Paradox.

Software para programar alarmas Paradox.

Amazon web service, alojamiento y servicios en nube.

Receptora virtual de eventos. terísticas de software como servicios $\mathrm{Saas}^{33}$; se diseñó y se implementó al crear un aplicativo software desarrollado en lenguaje Python ${ }^{34}$ versión 2.7 [28] con base de datos MysQL ${ }^{35}$ versión 5.0 [29]; está alojado en un servidor en la nube con servicio de conectividad y disponibilidad las 24 horas del día los 365 días del año. El sistema cuenta con una IP pública fija para la recepción de eventos provenientes de módulos GPRS ${ }^{36} \mathrm{e} \mathrm{IP}^{37}$ instalados en las alarmas, además de una aplicación móvil desarrollada con Android ${ }^{38}$ Studio [30] con el objetivo de interactuar con el sistema de alarma, generar emergencias de pánico y visualizar la totalidad de eventos recibidos por la plataforma.

El software de administración se estructuró en cinco secciones compuestas por "inicio", "usuarios", "abonados", "eventos" y "errores", tal como se evidencia en la figura 3.

Se incorporó infraestructura como servicio Laas $^{39}$ a través de la posibilidad de hacer uso futuro de receptoras de eventos físicas, además de vehículos aéreos no tripulados.

En el módulo 4, una empresa puede conectarse remotamente a través de un acceso web con el fin de monitorear sus abonados/clientes, y los usuarios finales pueden ver sus notificaciones con la aplicación móvil que se construyó inicialmente para el sistema operativo Android.

Una vez se genere un evento de alarma de robo, el dron realizará automáticamente un vuelo con el propósito de supervisar la casa monitoreada y capturar evidencias. Además, el propietario de la casa será notificado también de forma automática desde el sistema de administración frente a lo ocurrido en su vivienda a través de la aplicación móvil, correos electrónicos o con alertas al teléfono celular. El propietario tendrá la posibilidad de activar en su casa remotamente mecanismos tales como cierre de seguros o encendido de luces, y así mitigar la situación de emergencia desde su móvil.

Para la supervisión con drones, se seleccionó el dron Solo 3DR, un vehículo aéreo pequeño no

\footnotetext{
33 Software como servicios a través de la computación en la nube.

34 Lenguaje de programación.

Gestor de bases de datos relacionales.

Servicio general de paquetes vía radio.

Protocolo de Internet.

Sistema operativo en teléfonos celulares.

Infraestructura como, por ejemplo, servicios a través de la computación en la nube.
} 


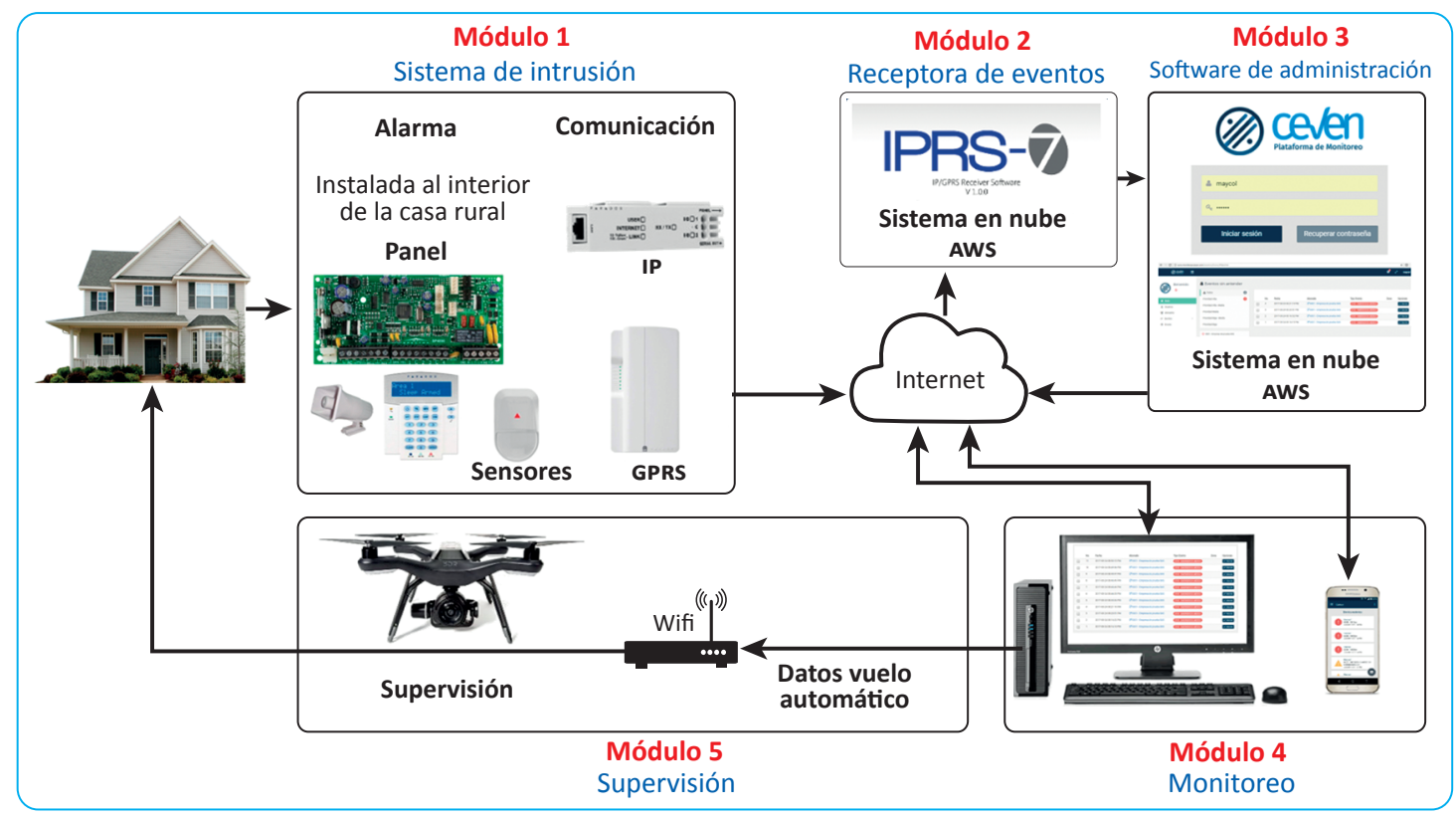

Figura 2. Implementación del prototipo de plataforma de vigilancia rural Fuente: elaboración propia

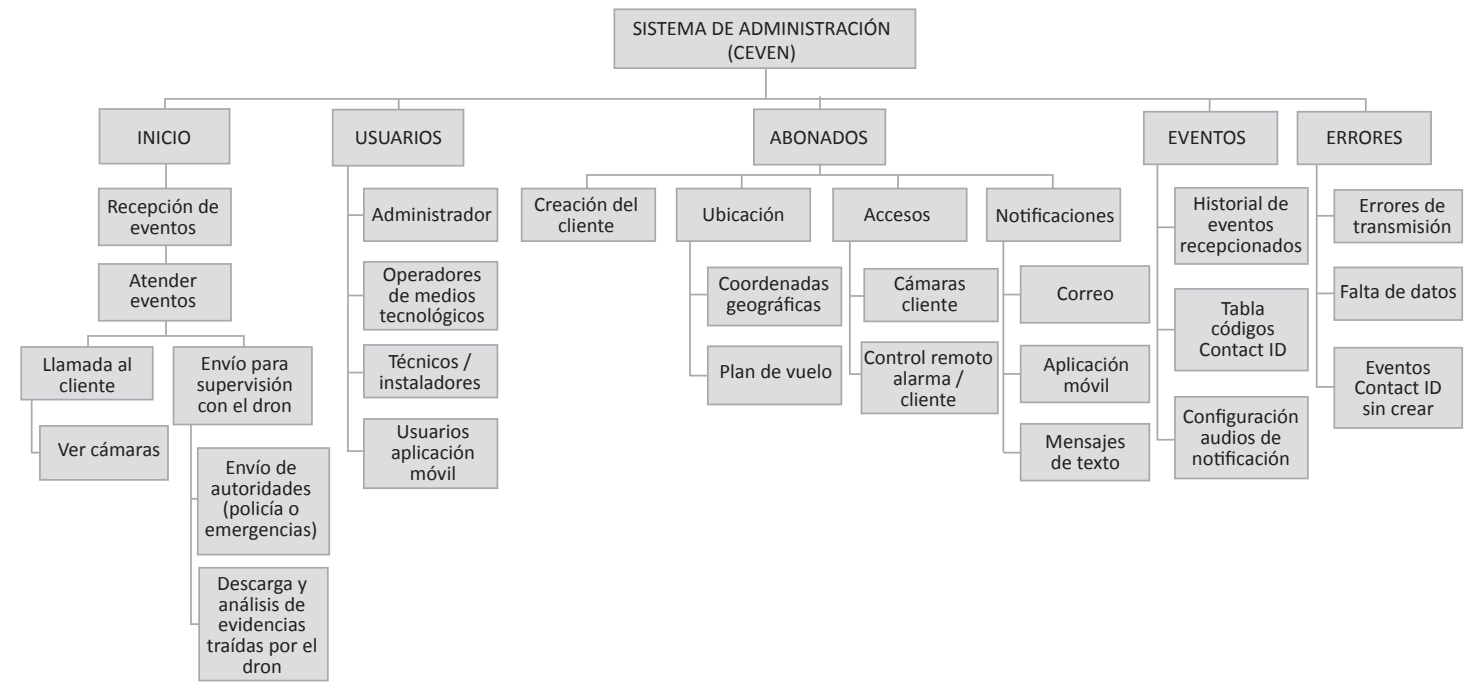

Figura 3. Estructura y secciones del software de administración Fuente: elaboración propia

tripulado que funciona gracias a cuatro motores y cuatro hélices. Las computadoras a bordo de Solo controlan la navegación, la altitud y las comunicaciones en el vuelo mientras envían telemetría y salida de video en tiempo real. Recibe entradas de control mediante una red wifi ${ }^{40}$ segura entre central de monitoreo en sitio y el dron.

40 Red inalámbrica 
A fin de establecer un vuelo automático, la plataforma envía las coordenadas geográficas del inmueble donde está ocurriendo la emergencia $y$, a su vez, le carga todo el plan de vuelo para el desplazamiento y los puntos de captura de fotos y toma de video.

La aplicación "3DR Solo" transmite video en vivo de una cámara Gopro ${ }^{41}$ a bordo a un computador o a un dispositivo Android o ios. La plataforma permite visualizar el video en vivo con una interfaz gráfica simplificada para controlar las funciones avanzadas de Solo. Además, la aplicación se conecta a la red 3DR Link para recibir salidas telemétricas y enviar entradas del control [31].

Los parámetros operativos más importantes del dron para realizar un vuelo se presentan en la tabla 1.

Tabla 1. Parámetros operativos dron solo

\begin{tabular}{ll}
\hline Tiempo de vuelo estimado & Hasta 25 minutos $^{*}$ \\
\hline Altitud máxima permitida & $\begin{array}{l}150 \text { pies }(46 \mathrm{~m}) \text { sobre } \\
\text { el nivel del piso }\end{array}$ \\
\hline Alcance & 5 millas \\
\hline Cap* $(8 \mathrm{~km})$
\end{tabular}

Fuente: [32]

Con el fin de permitir una interacción entre la plataforma creada y el dron, se utilizó DronKitPython $^{42}$ [33], el cual permite una conexión bidireccional para que la plataforma envíe las coordenadas geográficas, ordene el vuelo de forma automática y luego transfiera las imágenes y videos que el dron recopila en la supervisión al inmueble rural. DronKit-Python proporciona acceso mediante programación de telemetría y permite tanto la gestión de misiones como un control directo sobre el movimiento y las operaciones del vehículo aéreo.

41 Fabricante de videocámaras

42 Herramienta de desarrollo para programación de drones.
Es compatible con los vehículos que se comunican mediante el protocolo MAvLink ${ }^{43}$.

\section{Resultados}

Se construyó el software de administración de eventos y operación de supervisión con una interfaz gráfica intuitiva para el operador de medios tecnológicos, quien es la persona encargada del módulo de monitoreo con su acceso web; esta herramienta permite la visualización de todos los eventos recepcionados en tiempo real. El software permite crear cuentas de usuario en las que se almacena toda la información de los sistemas instalados y se discrimina el número de abonado ${ }^{44}$, las zonas de ubicación de sensores, el tipo de sensor por zona, el panel de alarma instalado, los usuarios del sistema, los horarios de activación/desactivación, el medio de comunicación, la visualización de las coordenadas geográficas en las que se encuentra instalada la alarma y el visor del vuelo del dron. En la figura 4, se presenta la interfaz gráfica como producto final del sistema de administración.

Cada vez que se recepciona un evento, se inicia un procedimiento de verificación y reacción, de manera que se da aviso automático a los clientes por medio de correos y por notificación a la aplicación móvil. En caso de requerir supervisión, el software entrega automáticamente las coordenadas y el plan de vuelo al dron para que se dirija hasta el inmueble y tome evidencias en imágenes y videos.

La plataforma se ha probado con diferentes sistemas de alarmas, entre ellos, Paradox DSC y Honeywell ${ }^{45}$, con sensores de movimiento y de fuego, comunicados por los equipos IP $150^{46} \mathrm{y}$ el $\mathrm{GPRS}^{47} \mathrm{LX}_{2} 0 \mathrm{~S},{ }^{48}$ de modo que ha recepcionado los eventos de alarma de forma correcta y sin retardos considerables. Como prueba inicial se le conectaron diez sistemas de alarma ubicados en el municipio de Villa de Leyva con la ayuda de la empresa Ecoss Ltda., a fin de monitorear el comportamiento de la plataforma con carga; su respuesta fue favorable debido a que no presentó bloqueos, notificó

\footnotetext{
43 Protocolo de comunicación para drones.

44 Cliente con un número de identificación de cuatro dígitos.

Fabricante de sistemas de seguridad.

Módulo de comunicación a través de Internet.

Servicio general de paquetes vía radio.

Módulo de comunicación vía red telefónica celular del

fabricante EBs.
} 


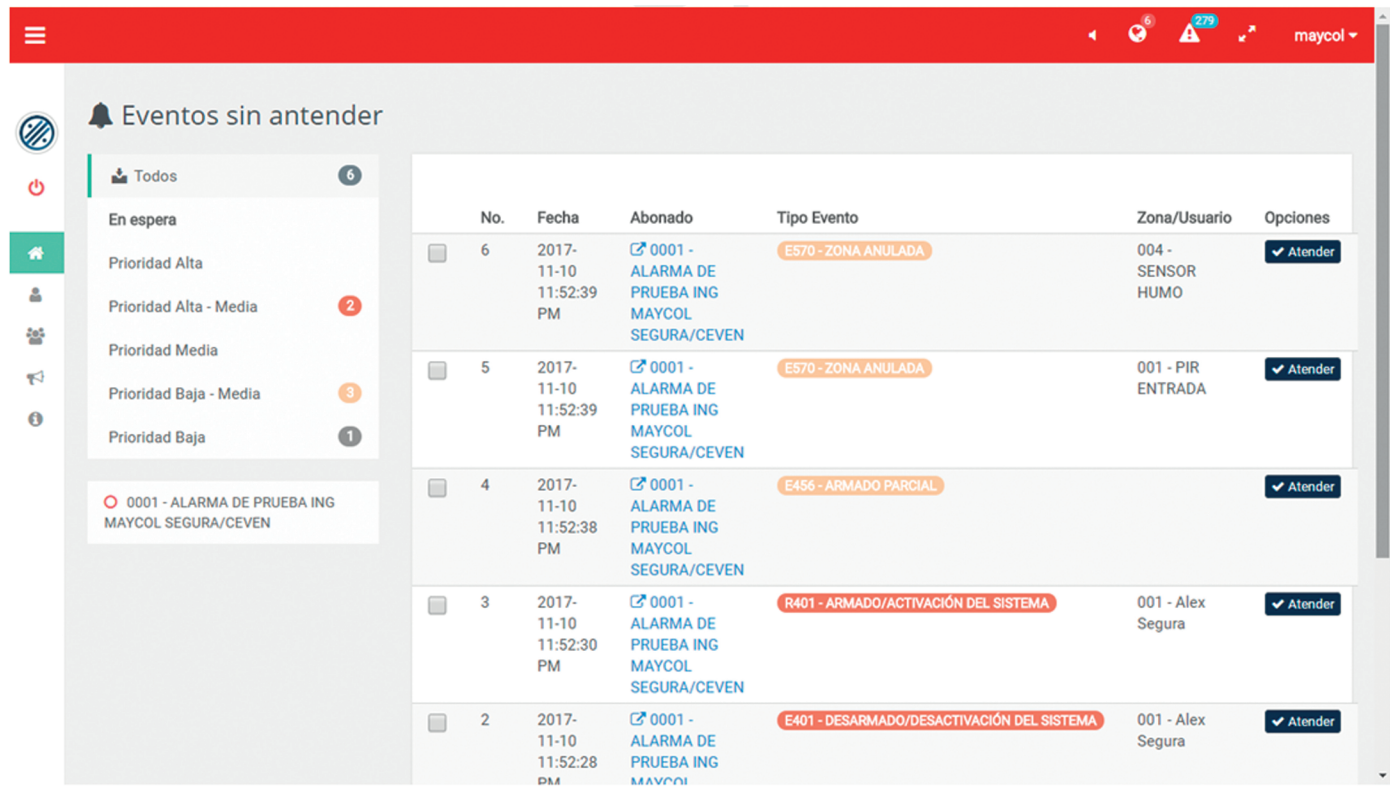

Figura 4. Interfaz-software de administración de abonados

Fuente: elaboración propia

los eventos de emergencia a la aplicación móvil y entregó la información al dron para el vuelo autónomo de supervisión.

Adicionalmente, se construyó una aplicación móvil con sistema operativo Android para usuarios finales, tecnicos instaladores y operadores de medios tecnológicos, la cual permite ver todos los eventos que emtite el sistema de alarma, tal como se ilustra en la figura 5.

Se realizaron pruebas entre envío de eventos por parte de la alarma con módulos de comunicación GPRS ${ }^{49}$ y la recepción en el software de administración, de lo que se obtuvo como resultado un retardo de dos segundos, en promedio, luego de 5000 eventos de pruebas. El mismo procedimiento se realizó con un módulo IP, lo que arrojó un tiempo promedio de un segundo.

En la supervisión con el dron, se encontró una respuesta rápida debido a que se planteó un escenario rural de prueba de acuerdo con las directrices de la Aeronáutica Civil Colombiana [34]. El dron se dispuso a 750 metros de distancia de donde se encontraba el inmueble monitoreado; se instaló una central de monitoreo a las afueras de la zona urbana del municipio de Villa de Leyva, la cual sirve como base del dron. Asimismo, se validó

49 Servicio general de paquetes vía radio.

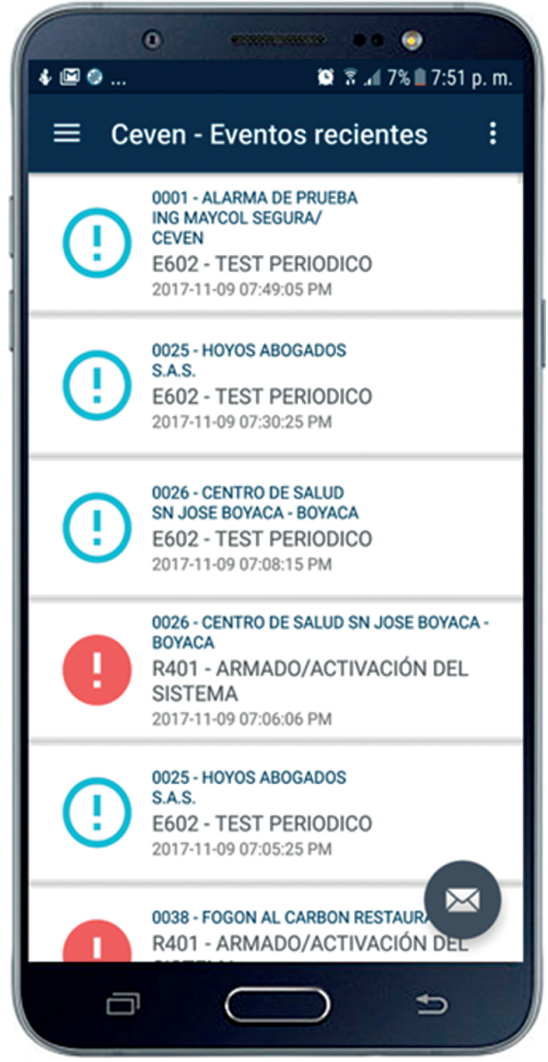

Figura 5. Interfaz aplicación móvil desarrollada Fuente: elaboración propia 
que es posible realizar supervisiones con drones de forma autónoma y reducir tiempos de respuesta ante incidencia de intrusión en zonas rurales; para esto se realizaron los cálculos que se presentan a continuación, para los cuales se tuvo como ejemplo la trayectoria de vuelo que se presenta en la figura 6 .

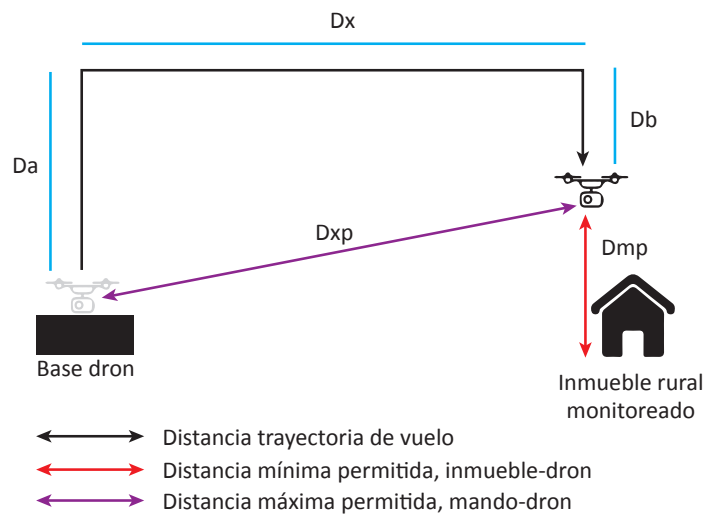

Figura 6. Trayectoria de vuelo del dron con distancias máximas según la Aerocivil Fuente: elaboraión propia

De acuerdo con la figura 6, hallamos las ecuaciones para el tiempo total de supervisión, el tiempo de desplazamiento entre base del dron e inmueble y el tiempo total en el vuelo del dron ida y vuelta con la captura de evidencias en fotos y videos

Ts = tiempo total de supervisión:

$$
T S=T d+T c
$$

Donde $T d=$ tiempo de desplazamiento y llegada al inmueble y $T c=$ tiempo de captura de imágenes.

${ }^{\star}$ Calculo del tiempo de desplazamiento " $T d$ ". De nuevo, a partir de la figura 6 concluimos que:

$T a=$ tiempo de ascenso, $D a=$ distancia de ascenso, $v a=$ velocidad de ascenso.

$T x=$ tiempo de desplazamiento, $\mathrm{Dx}=$ distancia de desplazamiento, $\mathbf{v x}=$ velocidad de desplazamiento.

$T b=$ tiempo de descenso, $D b=$ distancia de descenso, $v b=$ velocidad de descenso.

Luego:

$$
T d=T a+T x+T b
$$

Donde: $T a=D a / v a T x=D x / v x T b=D b / v b, y$ luego de reemplazar queda:

$$
T d=(D a / v a)+(D x / v X)+(D b / v b)
$$

Como el dron fue planeado para un vuelo automático, se programó con la velocidad crucero $^{50}$ dada por el fabricante; esta velocidad corresponde a $8 \mathrm{~m} / \mathrm{s}$ y es la que utilizará el vehículo aéreo tanto en ascenso y desplazamiento como en descenso, con lo cual se puede concluir que:

Velocidad crucero $=\mathrm{va}=\mathrm{vx}=\mathrm{vb}$.

$$
T d=(D a+D x+D b) /(\text { velocidad crucero })
$$

**Tiempo de captura de imágenes ${ }^{51}(T C)$ : se programó el dron para sobrevolar el inmueble durante cinco minutos, y en este tiempo se tomaron las evidencias en videos y fotos.

$$
T C=5 \mathrm{~min}
$$

${ }^{* * *}$ Tiempo total $(\mathrm{Tt})$ del dron para ir a una supervisión y regresar a su base.

$$
T t=(T d) 2+T S
$$

Para el caso práctico, realizó un vuelo con las especificaciones máximas permitidas en cuanto a distancias exigidas por la Aeronáutica Civil, es decir, $D x p=750 \mathrm{~m}, D a=152 \mathrm{~m}, D m p=50 \mathrm{~m}$. A fin de cumplir con esto se establecieron los siguientes parámetros:

$D x=750 \mathrm{~m}$

$D b=102 \mathrm{~m}$

$T C=5$ min (tiempo de captura de imágenes programada en el dron).

De la ecuación 3, se calculó el tiempo de desplazamiento:

50 Velocidad ejercida en una trayectoria rectilínea horizontal, la velocidad crucero la da el fabricante del dron.

51 Tiempo indicado por la empresa de seguridad que ayudo en la implementación del prototipo. 
$T d=(D a+D x+D b) /($ velocidad crucero $)$

$T d=(152 \mathrm{~m}+750 \mathrm{~m}+102 \mathrm{~m}) /(8 \mathrm{~m} / \mathrm{s})$

$\mathrm{Td}=125,5 \mathrm{~s}=2,09 \mathrm{~min}$ (este sería el tiempo que demora el dron en llegar al inmueble).

Después, de la ecuación 1, se calculó el tiempo total de supervisión:

$T s=T d+T c$

Donde $T c=5 \mathrm{~min}$

$T s=2,09 \mathrm{~min}+5 \mathrm{~min}$

$T s=7,09$ min (tiempo que tardaría el dron en desplazarse al inmueble y sacar imágenes durante 5 $\min )$.

A fin de calcular el tiempo total (Tt) que le toma al dron ir a la supervisión y regresar a su base se aplicó la ecuación 4 .

$T t=(T d) 2+T s$

$T t=(2,09 \mathrm{~min}) * 2+5 \mathrm{~min}=9,18 \mathrm{~min}$

Para identificar que cumplimos con la Dxp, se realizó el siguiente calculo:

$$
\begin{aligned}
& D x p=\sqrt{D x^{2}-D m p^{2}} \\
& D x p=\sqrt{750 m^{2}-50 m^{2}}=748,33 \mathrm{~m}
\end{aligned}
$$

Con el propósito de establecer un vuelo automático, la plataforma envía al dron las coordenadas geográficas del inmueble donde ocurre la emergencia en ese momento, y a su vez le carga toda la ruta para el desplazamiento y los puntos de captura de fotos y toma de video. La plataforma permite visualizar el video en vivo con una interfaz gráfica simplificada para controlar las funciones avanzadas del dron Solo. Además, la aplicación se conecta a la red 3DR Link con el fin de recibir salidas telemétricas y enviar entradas del control.

Entre las características empleadas para el vuelo autónomo del dron, se destacan la posibilidad de conectarse a partir de un código comando, obtener y establecer la información de estado del dron, el envío de mensajes personalizados para controlar el movimiento y crear misiones en modo automático.

La plataforma de monitoreo de alarmas está compuesta por un sistema en la nube que podría prestar un servicio para empresas de seguridad electrónica pues permite monitorear instalaciones de forma directa, sin intermediarios y sin invertir en infraestructura tecnológica. La suma de Saas más Laas es lo que permitió caracterizar todo el proyecto como una plataforma.

$\mathrm{Al}$ realizar una comparación entre la plataforma desarrollada y los sistemas de monitoreo convencional, se puede resaltar que la plataforma ofrece características adicionales en lo tecnológico, en servicios y en lo económico, según se establece en la comparación de la tabla 2.

\begin{tabular}{|c|c|c|c|}
\hline Aspecto & Descripción & $\begin{array}{c}\text { Sistema } \\
\text { convencional }\end{array}$ & $\begin{array}{c}\text { Plataforma } \\
\text { desarrollada }\end{array}$ \\
\hline \multirow{3}{*}{ Tecnológico } & Computación en la nube & & $\mathrm{X}$ \\
\hline & Uso de drones & & $\mathrm{X}$ \\
\hline & Comunicación vía GPRS e IP & $\mathrm{X}$ & $\mathrm{X}$ \\
\hline \multirow{5}{*}{ Servicios } & Mensajería de correo electrónico y texto automática & $\mathrm{X}$ & $\mathrm{X}$ \\
\hline & Internet con IP pública fija & $\mathrm{X}$ & $\mathrm{x}$ \\
\hline & Supervisión con drones & & $\mathrm{x}$ \\
\hline & Supervisión motorizada & $\mathrm{x}$ & \\
\hline & Monitoreo-administración remota & & $\mathrm{x}$ \\
\hline \multirow{2}{*}{ Económico } & Inversión inicial & $\mathrm{x}$ & \\
\hline & Costos por actualizaciones en el sistema administrador del monitoreo & $\mathrm{x}$ & \\
\hline
\end{tabular}

Tabla 2. Comparación-plataforma de monitoreo vs. modelos convencionales

Fuente: elaboración propia 


\section{Discusión}

Dada la solución inicial para reducir tiempos de respuesta en la supervisión en zonas rurales, se puede plantear un posible modelo de negocio aceptado por las empresas de monitoreo actuales, razón por la cual se establece un análisis DOFA (debilidades, oportunidades, fortalezas y amenazas) para la plataforma desde una prospectiva empresarial (tabla 3).

Dentro del ámbito de la seguridad electrónica, podrían surgir preguntas tales como: ¿para qué un dron supervisando si se pueden instalar cámaras en el inmueble rural y captar las evidencias? Las cámaras de seguridad son un complemento a los sistemas de alarma: uno detecta y el otro graba; sin embargo, para el caso de estudio en zonas rurales no es viable decir que las cámaras sean una opción, en primer lugar porque no se consigue Internet banda ancha que permita tener un acceso remoto al videograbador y, en segundo lugar, porque al detectar sistemas de seguridad en medio de un hurto los ladrones tienden a destruir o llevarse las cámaras y las videograbadoras para no dejar evidencia.

¿El dron viola la intimidad de las personas cuando realiza la supervisión? La intimidad es un tema sensible con el uso de drones a nivel mundial en razón a la facilidad con la que una persona puede capturar imágenes sin permiso en áreas privadas. No obstante, el prototipo de plataforma planteado debe respetar las distancias de sobrevuelo que determina la Aeronáutica Civil y sobrevolara a una

Tabla 3. Matriz DofA-plataforma de monitoreo

\section{Análisis DOFA}

1. Prototipo de plataforma para vigilancia de inmmuebles rurales usando computación en la nube y supervición con drones.
Oportunidades

1. El desarrollo tecnológico y la innovación en productos software para diferentes tipos de monitoreo (alarmas, personas, carros).

2. La apertura de nuevos mercados en el campo de la seguridad electrónica.

3. Nuevos inversionistas para implementar a gran escala el proyecto.

\section{Fortalezas}

1. Supervisión innovadora con drones.

2. Sistema $100 \%$ en nube.

3. Plataforma multiusuario.

4. Continuidad del negocio para cualquier empresa de monitoreo.

5. Disponibilidad del servicio 24/7.

6. Acceso a usuarios finales a través de aplicaciones móviles.

7. Presta todas las herramientas de una central de monitoreo.

\section{Estrategias Fo}

1. Desarrollo de nuevas herramientas software de acuerdo con las necesidades de los clientes.

2. Iniciar la promoción de la plataforma a nivel nacional e internacional.

3. Búsqueda de socios inversionistas para ampliar el mercado.
Amenazas

1. Llegada de competidores.

2. Empresas que incorporen la idea de supervisar con drones con sus plataformas actuales.

3. Pérdida de mercado.

\section{Debilidades}

1. Dependencia de un tercero para alojar el sistema en nube.

2. Condiciones ambientales para los vuelos del dron en tareas de supervisión.

3. Regulación de la aeronáutica civil colombiana para vuelos con drones sobre zonas urbanas con fines económicos.

4. Desconocimiento en el uso de la plataforma por parte de los clientes.

\section{Estrategias Do}

1. Solicitar ante la Aeronáutica Civil la revisión de la regulación para permitir la operación en zonas urbanas y rurales, teniendo en cuenta que esta puede beneficiar a los entes del Estado como la Policía y los Bomberos.

2. Planear la capacitación a posibles clientes sobre uso y manejo de la plataforma de forma presencial y a través de plataformas virtuales de aprendizaje como Moodle.

\section{Estrategias FA}

1. Diseñar un programa dentro de la plataforma para el servicio al cliente con la finalidad de mantener su fidelidad.

2. Planear estrategias de marketing para impulsar la competencia e incorporación al mercado.

3. Diseñar un plan de seguimiento al desempeño de funcionamiento de la plataforma. 
altura mínimo de $50 \mathrm{~m}$. Por otro lado, las empresas de seguridad que llegaran a utilizar la plataforma deben informar al cliente final sobre el tipo de supervisión y pedir un permiso de sobrevuelo.

\section{Conclusiones}

Por medio de pruebas de campo, se midieron los tiempos de respuesta entre el evento enviado por un sistema de alarma que está ubicado en Villa de Leyva y la recepción de dicho evento en el software de administración alojado en un servidor ubicado en Norte América. Estas pruebas arrojaron un resultado que indica cómo un evento o señal de alarma tarda aproximadamente 1,5 segundos, en promedio, con el empleo de módulos de comunicación GPRS e IP. Con este periodo de tiempo encontrado se valida la rápida respuesta del sistema de seguridad entre emisor y receptor.

Se identifica como aporte principal del software de administración la posibilidad que brinda de interactuar directamente con los vehículos aéreos no tripulados, así como realizar tareas programadas o dirigidas para la supervisión gracias a la programación que se desarrolló con el lenguaje Python que interactúa con el protocolo MavLink a través del envío de información en una red inalámbrica de 2,4 GHz.

El tiempo de supervisión del dron calculado matemáticamente en comparación con la prueba práctica arrojó que existe un margen de diferencia de aproximadamente el $10 \%$ por encima o por debajo, debido a la velocidad del viento. Si el dron vuela en contra del viento tarda más, o si va en la misma dirección puede llegar más rápido de lo calculado.

Se logró solucionar la problemática inicial de reducir tiempos de respuesta en la supervisión para el servicio de monitoreo rural al calcular que, por cada 750 metros, el dron tarda 2,09 minutos a una velocidad de $8 \mathrm{~m} / \mathrm{s}$, y la supervisión con captura de imágenes tarda 7,09 min. No obstante, la plataforma en su módulo cinco queda delimitada a condiciones ambiente favorables para que el dron pueda volar de forma óptima. De igual manera, se establece un nuevo modelo de negocio al integrar tecnología a nivel de instrumentación, comunicaciones, computación en la nube y vehículos aéreos no tripulados.
Es viable poner en operación la plataforma para ambientes reales de monitoreo de alarmas dada su comprobación y, además, a la demanda que existe en razón a la cantidad de hurtos que se presenta en zonas rurales colombianas. De esta manera queda para uso en zonas rurales y se excluye su uso en zona urbana por regulación de la Aeronáutica Civil sobre uso de drones.

La planificación de vuelo fue algo indispensable a la hora de enviar el dron a una misión de supervisión a fin de validar la plataforma planteada. Se dejó la opción para que permita vuelos autónomos programados y la opción de que sea un operador quien dirija el vuelo. Los motivos surgieron por las variables ambientales que afectan los vuelos y que solo un humano puede detectar de forma autónoma o con instrumentación. Estas variables son la velocidad del viento, temperatura exterior y lluvia. Adicionalmente, para enviar un dron a supervisión se debe realizar una evaluación minuciosa del terreno e identificar los obstáculos.

El proyecto muestra una aplicación de los drones en Colombia limitada por la normativa existente de la Aeronáutica Civil, la cual permite desplazamientos máximos de $750 \mathrm{~m}$ entre mando y dron y prohíbe vuelos autónomos. Sin embargo, el sistema diseñado puede ser una herramienta de ayuda a entidades como la Policía, los Bomberos y los centros de reacción como mecanismo primario de verificación de incidencias en hurtos, incendios, eventos médicos, monitoreo del tránsito vehicular y catástrofes naturales. No obstante, la plataforma puede llegar a ser empleada en otros países donde los drones se regulen para realizar labores con fines comerciales y de control.

La plataforma de monitoreo en la nube proporciona facilidades de acceso a la prestación del servicio a empresas de monitoreo porque no tienen que hacer una inversión inicial en infraestructura y en software, además de garantizar la seguridad de la información, la continuidad del negocio y la disponibilidad del servicio las 24 horas del día. Adicionalmente, la plataforma queda abierta a incorporar señales de varios tipos de sensores diferentes a los "on/off" con la inclusión de conversores analógicos a digital e involucrar nuevas áreas del conocimiento en las que trabaje en el monitoreo remoto de diferentes variables físicas.

En fin, se trabajó con receptoras virtuales de eventos con el fin de recepcionar señales de alarma trasmitidas por módulos de comunicación IP/GPRS 
y se comprobó que estas herramientas software funcionan bien al simular las receptoras físicas convencionales; esto reduce costos considerables y permite una operación en nube con facilidad de acceso a los servicios de monitoreo.

\section{Referencias}

[1] Supervigilancia, "Empresas de monitoreo/seguridad sin armas-servicios vigentes", Supervigilancia.gov.co, p. 1, 2017. [En línea]. Disponible en: http://www.supervigilancia.gov.co/?idcategoria $=6846418 \&$ download $=\mathrm{Y}$

[2] M. Segura y J. Mesa, "Modelo de monitoreo de alarmas con uso de la nube y supervisión apoyada con drones", en Memorias ponencia Congreso Internacional de Ciencias Básicas e Ingeniería, Unillanos CICI: 2016, p. 2. [En linea]. Disponible en: http://fcbi. unillanos.edu.co/cici/Articulos/CICI_2016_paper_124.pdf

[3] J. Ricardo y B. Cubides, "Registros de la criminalidad en Colombia y actividad operativa de la Policía Nacional durante el año 2015", Revista Criminalidad, vol. 58, pp. 10-12, 2016. [En línea]. Disponible en: https://www.policia.gov.co/file/37281/download? token $=\mathrm{QmKmroOR}$

[4] F. R. Jiménez-López, "Prototipo de sistema de vigilancia de ganado usando red de supervisión inalámbrica para prevención de abigeato", Revista Ingenio Magno,vol.7,no.2,pp.89-95,2016. [Enlínea].Disponible en: http://revistas.ustatunja.edu.co/index.php /ingeniomagno/article/view/1196/1167

[5] Monitoreo.com, "Monitoreo-plataforma de alarmas en nube," Monitoreo.com, p. 1, 2017. [En línea]. Disponible en: https://www. monitoreo.com/

[6] Keitech, "Soluciones integrales de software," Keitechcolombia.com, 2017, p. 1. [En línea]. Disponible en: http://keitechcolombia.com/web/

[7] A. M. M. Peillard, "Vigilancia en la modernidad tardía: el monitoreo telemático de infractores”, Revista Política Criminal, vol. 8, pp. 408-471, 2013. [En línea]. Disponible en: http://www.scopus.com/ inward/record.url?eid=2-s2.0-84892597519\&partnerID=tZOtx3y1

[8] Y. Q. Han, L. Ma y H. L. Guo, "Design of monitoring alarm system based on GPRS MMs", Advanced Materials Research, vol. 1049-1050, pp. 2149-2152, 2014. [En línea]. Disponible en: http://www.scopus.com/ inward/ record.url?eid=2-s2.0-84920691839\&partnerID=tZOtx3y1

[9] L. Ma, T. Yuan, F. Xia, M. Xu, J. Yao y M. Shao, “A high-confidence cyber-physical alarm system: De- sign and implementation", Proceedings-2010 IEEE/ ACM International Conference on Green Computing and Communications, GreenCom 2010, 2010 IEEE/ ACM International Conference on Cyber, Physical and Social Computing, CPSCom 2010: 2010, pp. 734-738. [En línea]. Disponible en: http://ieeexplore.ieee.org/document/5724880/?reload=true

[10] J. Moreno, "Sistema de monitoreo, vigilancia y control mediante el uso de drones para los campos agrícolas", tesis de grado, Universidad Autónoma Regional de los Andes, pp. 30-95, 2015. [En línea]. Disponible en: http://dspace.uniandes.edu.ec/bitstream/123456789/1636/1/TUTSIS005-2015.pdf

[11] G. Galdon-Clavell, "Si la videovigilancia es la respuesta, ¿cuál era la pregunta? Cámaras, seguridad y políticas urbanas 1", Revista EURE (Santiago), vol. 41, pp. 81-101, 2015. [En línea]. Disponible en: http://www.scielo.cl/scielo.php?script=sci_arttext\&pid=S0250-71612015000300004

[12] Ministerio de Defensa de España, "Los sistemas no tripulados", Documentos de seguridad y defensa, pp. 10-134, 2012. [En línea]. Disponible en: https:// publicaciones.defensa.gob.es/media/downloadable/ files/links/P/D/PDF223.pdf

[13] S. E. G. Palacios, "Ingeniería básica para el diseño sistema de monitoreo, detección y alarma, humo, fuego y gas (F\&G) de las estaciones de compresión de gas de la Transportadora de Gas Internacional TGI", proyecto de grado, Universidad Pontificia Bolivariana, pp. 14-74, 2011. [En línea]. Disponible en: https://repository.upb.edu.co/bitstream/handle/ 20.500.11912/1288/digital_20465.pdf?sequence=1

[14] J. Kleinschmidt, "Drones y el orden legal internacional. Tecnología, estrategia y largas cadenas de acción, Revista Colombia Internacional, vol. 84, pp. 17-42, 2015. [En línea]. Disponible en: http://www. scielo.org.co/pdf/rci/ n84/n84a02.pdf

[15] B. R. Christensen, "Use of UAV or remotely piloted aircraft and forward-looking infrared in forest, rural and wildland fire management: evaluation using simple economic analysis", New Zealand Journal of Forestry Science, vol. 45, pp.1-16, 2015. [En línea]. Disponible en: http://www.scopus.com/inward/ record.url?eid=2-s2.0-84940984484\&partnerID=tZO tx3y1

[16] L. Tang y G. Shao, "Drone remote sensing for forestry research and practices", Journal of Forestry Research, vol. 16, pp. 2-8, 2015. [En línea]. Disponible en: https://link.springer.com/article/10.1007/s1167 6-015-0088-y

[17] L. Di Puglia Pugliese, F. Guerriero, D. Zorbas y T. Razafindralambo, "Modelling the mobile target covering problem using flying drones", Revista Optimization Letters, vol. 10, pp. 1021-1052, 2015. 
[En línea]. Disponible en: http://www.scopus.com/ inward/record.url?eid=2-s2.0-84939636075\&partnerID=tZOtx3y1

[18] D. Floreano y R. J. Wood, "Science, technology and the future of small autonomous drones", Revista Nature, vol. 521, pp. 460-466, 2015. [En línea]. Disponible en: https://www.nature.com/articles/nature 14542

[19] M. R. Calo, "The drone as privacy catalyst", Revista Stanford Law Review, vol. 29, pp. 29-33, 2011. [En línea]. Disponible en: https://cyberlaw.stanford. edu/files/publication/files/64-SLRO-29.pdf

[20] A. Kharsansky, "Diseño e implementación de un sistema embebido de control de actitud para aeronaves no tripuladas", tesis de grado, Universidad de Buenos Aires, pp. 1-106, 2013. [En línea]. Disponible en: http://laboratorios.fi.uba.ar/lse/tesis/LSEFIUBA-Tesis-Grado-Alan-Kharsansky-2013.pdf

[21] R. A. Zahawi, J. P. Dandois, K. D. Holl, D. Nadwodny, J. L. Reid y E. C. Z. Ellis, "Using lightweight unmanned aerial vehicles to monitor tropical forest recovery", Biological Conservation, vol. 186, pp. 287 295, 2015. [En línea]. Disponible en: http://www.scopus.com/inward/record.url? eid=2-s2.0-849276708 51\&partnerID=tZOtx3y1

[22] Organización de Aviación Civil Internacional. Sistemas de aeronaves no tripuladas (UAS). Montréal: Organización de Aviación Civil Internacional, 2011, pp. 1-56. [En línea]. Disponible en: http://goo.gl/ yMxnLn

[23] Ademco. Digital Communication Standard-Ademco Contact ID Protocol-for alarm system communications. 1999, pp. 1-27. [En línea]. Disponible en: http://www.technoimport.com.co/Producto/pdfs/ ADEMCO-DC05_Contact_ID.pdf

[24] Paradox. SP4000. 2010, pp. 1-32. [En línea]. Disponible en: http://www.paradox.com/Downloader $/$ ID $=3158$
[25] Paradox. Modulo GPRS PCS3o. 2011, pp. 1-16. [En línea]. Disponible en: http://www.paradox.com/ Downloader/?T=URL\&URL=/Documentation/ Manuals/ARCHIVES_InstallationProgramming/ PCS300-EU02.pdf

[26] Paradox. Modulo IP150. 2015 pp. 1-2. [En línea]. Disponible en: http://www.paradox.com/Downloader/?ID=7040

[27] Amazon, "Amazon elastic compute cloud", Amazon. com, 2017. [En línea]. Disponible en: https://goo.gl/ voGzZA

[28] Python Organización, "Lenguaje de programación," Python.org, 2016. [En línea]. Disponible en: https:// www.python.org/

[29] Oracle Corporation, "Bases de datos MysquC", Mysql.com, 2016, p. 1. [En línea]. Disponible en: https://www.mysql.com/

[30] Android Developers, "Programación en Android para dispositivos móviles", Android.com, p. 1, 2017. [En línea]. Disponible en: https://developer.android.com/about/android.html

[31] 3DR, “3D Robotics,", 3dr.com, 2017, p. 1. [En línea]. Disponible en: https://3dr.com/solo-drone/

[32] 3DR. Solo-Manual de usuario, "Parámetros operativos dron solo”, Berkeley, CA: 3D Robotics, 2017. [En línea]. Disponible en: https://3dr.com/wp-content/ uploads/2017/03/Solo_User_Manual_v1_Spanish. pdf

[33] 3DR DroneKit-Python, "DroneKit-Python," Dronekit.io, p. 1, 2015. [En línea]. Disponible en: http:// python.dronekit.io/about/overview.html

[34] AerocivilColombiana, "Requisitosgenerales deaeronavegabilidad y operaciones para RPAs en Colombia," Circular Reglamentaria, no. 002,p. 8,2015. [Enlínea]. Disponible en: http://www.aerocivil.gov.co/normatividad/_layouts/15/WopiFrame.aspx?sourcedoc=/ normatividad/Resoluciones \%202015/CIRCU LAR\%20REGLAMENTARIA\%20\%20002\%20-\%20 RPAS.pdf\&action=default 